\title{
Nanomateriales con actividad microbicida: una alternativa al uso de antibióticos
}

\author{
Roberto Vazquez-Muñoz, ${ }^{*}$ Alejandro Huerta Saquero*
}

RESUMEN: Las enfermedades infecciosas representan un gran problema a nivel mundial, y consumen una cantidad importante de recursos públicos y privados. Las infecciones más comunes son las virales y las bacterianas, seguidas por las fúngicas. Actualmente, se utilizan agentes antibióticos o antivirales para combatir las enfermedades infecciosas, pero existen muchos problemas relacionados con su uso, tales como la aparición de microrganismos resistentes y el complicado proceso para crear nuevas sustancias. La bionanotecnología puede ayudar a resolver el problema de las enfermedades infecciosas; un ejemplo es mediante el uso de las nanopartículas de plata. Éstas tienen propiedades clínicas importantes, particularmente como antibióticos y antivirales. Por lo tanto, es necesario investigar las interacciones y efectos que existen entre las nanopartículas de plata en los microrganismos y en el ser humano, para desarrollar nuevas terapias en la medicina.

PALABRAS CLAVE: bionanotecnología - enfermedades infecciosas - nanopartículas de plata.

ABSTRACT: Infectious diseases are a major problem worldwide, and consume a significant amount of public and private resources. The most common infections are viral and bacterial, followed by fungal infections. Currently, antibiotics or antiviral agents are used to combat infectious diseases, but there are many problems associated with their use, such as the emergence of resistant organisms and highly complicated process to create new substances. Bionanotechnology can help to solve the problem of infectious diseases; an example is by using silver nanoparticles. These have important clinical properties, particularly as antibiotics and antivirals. Therefore, it is necessary to investigate the effects and interactions of the silver nanoparticles in microorganisms and in human cells, in order to develop new therapies in medicine.

KEYWORDS: bionanotechnology - infectious diseases - silver nanoparticles.

\section{Enfermedades infecciosas}

Las enfermedades infecciosas se definen como la manifestación clínica consecuente a una infección provocada por un agente patógeno, generalmente virus o microrganismos, como las bacterias y los hongos. Estas enfermedades representan uno de los problemas de salud más relevantes a nivel mundial, debido a su impacto social y económico (Scott, 2009) y se encuentran entre las principales causas de muerte en el mundo. Además, tanto su incidencia como su número (enfermedades emergentes) se incrementan con el paso del tiempo (NLM, 2013; WHO, 1997). Las primeras causas de estas enfermedades son los virus y las bacterias, seguidas por los hongos.

* Departamento de Bionanotecnología, Centro de Nanociencias y Nanotecnología, UNAM. 
Las enfermedades infecciosas tienen un gran impacto a nivel social y económico. Su relevancia es tal, que uno de los "Objetivos del Desarrollo del Mileno" de la Organización de las Naciones Unidas, involucra el combate de estas enfermedades (WHO, 2000). En Estados Unidos, el costo para combatirlas es de 120 mil millones de dólares al año (NIAID, 2009); mientras que la tuberculosis, por sí sola, le cuesta al mundo 12 mil millones de dólares al año. Otras, como el VIH/SIDA, consumen el $12 \%$ del producto interno bruto de los países africanos subsaharianos (Fonkwo, 2008).

La mayoría de las muertes por enfermedades infecciosas son ocasionadas por unas pocas especies de microrganismos. Entre las enfermedades infecciosas mortales destacan la neumonía, la tuberculosis, las enfermedades diarreicas, el paludismo, el sarampión y el VIH/SIDA. En conjunto, estas provocan más de la mitad de todas las defunciones prematuras en el mundo, causando sobre todo la muerte de niños y adultos jóvenes, particularmente en países en desarrollo (UNICEF/WHO, 2009).

\section{Las infecciones bacterianas}

Las bacterias son una de las principales causas de muerte por enfermedades infecciosas, tales como la neumonía, la tuberculosis y las enfermedades diarreicas (UNICEF/WHO, 2009). Un rasgo distintivo de estos agentes causales es el surgimiento de cepas multirresistentes a los antibióticos que tradicionalmente se emplean para su control y eliminación. En la figura 1, se muestran algunos ejemplos de bacterias patógenas.

La plasticidad genómica de los microrganismos y sus eficientes mecanismos de transmisión de información genética vía horizontal (que se transmiten de una bacteria a otra de la misma o de diferente especie) permiten la

FIGURA 1. Ejemplos representativos de bacterias patógenas: a) Helicobacter pylori, y b) Mycobacterium tuberculosis.

(a)

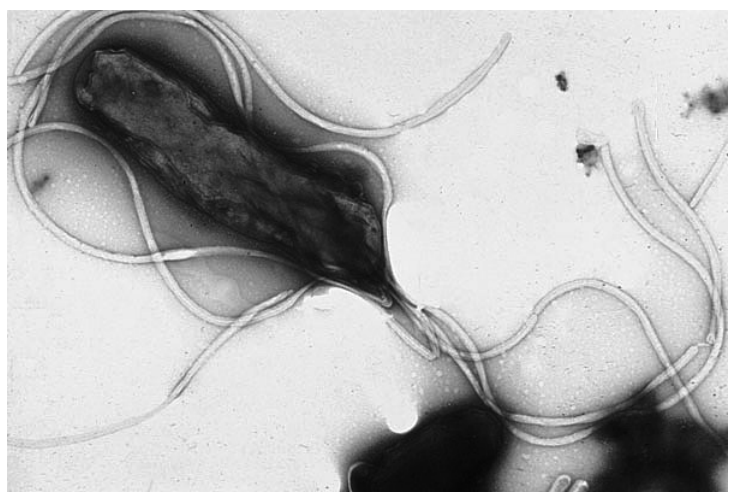

(b)

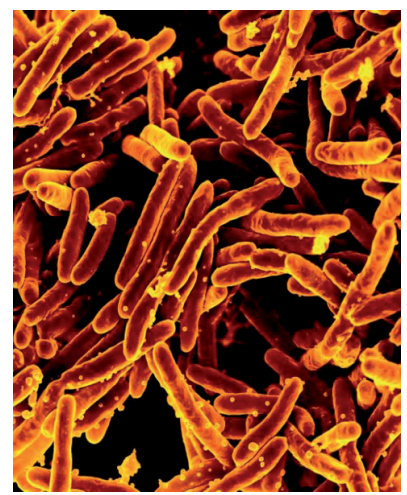

Fuente: Imágenes obtenidas de (a) Wiki commons (Tsutsumi Y.) y (b) NIAID (en flickr.com). 
adquisición de nueva información genética (Ulrich et al., 2004). Esta plasticidad favorece la supervivencia bacteriana, principalmente cuando se comparten genes que confieren resistencia a antibióticos, metales pesados o que favorecen el crecimiento en condiciones extremas, tales como la desecación, altas o bajas temperaturas, etc. Esto ha favorecido que resurjan enfermedades infecciosas o que aparezcan nuevas.

\section{Enfermedades causadas por hongos: micosis}

Las micosis tienen mayor incidencia en personas inmunocomprometidas, aunque también como infecciones nosocomiales (Fridkin, 2005) y en personas sanas. Desde principios de 1980, los hongos son una de las principales causas de las infecciones humanas (McNeil et al., 2001), y en algunos países su incidencia se ha incrementado en más del 200\% (Martin et al., 2003). Los factores que han contribuido al incremento de las infecciones fúngicas son muy diversos, entre los que destacan: el incremento de la población inmunocomprometida; uso inadecuado de agentes antibióticos, quimioterapias citotóxicas, surgimiento de cepas patógenas no comunes, e, inclusive, la globalización y la migración, que ayudan a diseminar cepas patógenas exóticas (Walsh et al., 2004; WHO, 2008).

El género Candida, agente causal de la candidiasis, es el más representativo de las micosis, y es la primera causa de las infecciones fúngicas en el mundo (Eggimann et al., 2003). La especie más común del género es $C$. albicans, levadura dimórfica (cambia de forma, figura 2a), muy versátil y con una gran plasticidad genómica (Clancy y Nguyen, 2012). Otras infecciones fúngicas importantes son: aspergilosis, blastomicosis, coccidioidomicosis, histoplasmosis y esporotricosis, además de las causadas por las especies de Cryptococcus (NIAID, 2011). Algunas micosis han incrementado su incidencia, tales como las causadas por $C$. glabrata, A. terreus, y otros mohos noAspergillus. Mientras que, entre las infecciones emergentes, destacan levaduras oportunistas como Trichosporon y Rhodotorula, y zigomicetos como Fusarium y Scedosporium (Pfaller et al., 2006). En la figura 2 se observan algunos hongos patógenos representativos.

\section{Otras infecciones: virus y protozoarios}

Otras enfermedades infecciosas importantes son las causadas por virus y los protozoarios. Los virus se forman a partir de material genético recubierto por una cápsula. Son muy pequeños y no son afectados por los antibióticos. Para combatirlos se requiere el uso de antivirales. Los virus causan enfermedades infecciosas conocidas como el resfriado común, la gripe y las verrugas. También causan enfermedades graves como el VIH / SIDA, la viruela y las fiebres hemorrágicas. (NLM, 2014). Según la Organización Mundial de la Salud (WHO, 2013), más de 530 millones de personas tienen el virus que causa el 
FIGURA 2. Ejemplos representativos de hongos patógenos: a) Candida albicans (hifas, pseudohifas y blastoconidios); b) Aspergillum spp (conidióforo), y, c) Fusarium spp (conidióforos y macroconidios).

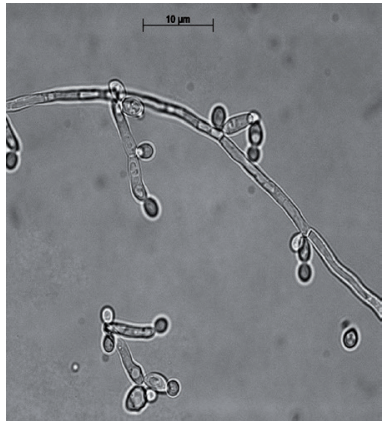

(a)

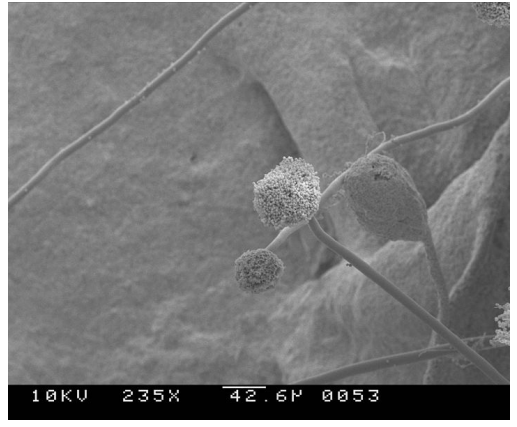

(b)

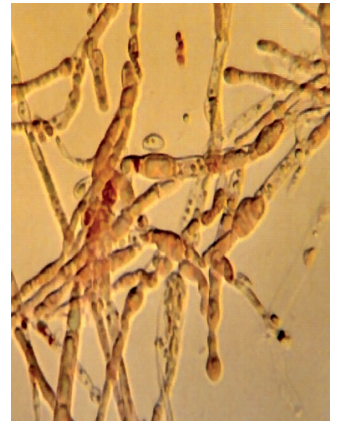

(c)

Fuente: Imágenes obtenidas de (a) Roberto Vazquez-Muñoz; (b) y (c) Wiki commons.

herpes genital (HSV-2), más de 290 millones de mujeres están infectadas por el virus del papiloma humano (VPH). Además, algunas enfermedades infecciosas de transmisión sexual pueden aumentar el riesgo de contraer el VIH.

Los protozoos son organismos microscópicos eucariotas unicelulares, que pueden ser de vida libre o de naturaleza parasitaria. La transmisión de protozoos que viven en un intestino humano a otro ser humano se produce normalmente a través de una vía fecal-oral (alimentos o agua contaminados, por ejemplo), mientras que los protozoos que viven en la sangre o en los tejidos de los seres humanos se transmiten a otros seres humanos por un vector. Los protozoos que son infecciosos para los seres humanos se pueden clasificar en cuatro grupos: Sarcodina (como la amiba), Mastigóforos (Giardia, Leishmania), Cilióforos (Balantidium) y Sporozoa (Plasmodium, Cryptosporidium) (CDC, 2010). Entre las infecciones más comunes destacan la amibiasis, giardiasis, la enfermedad del sueño, la leishmaniasis, la toxoplasmosis y la malaria (Med-health, 2014).

\section{Antibióticos}

Los agentes antibióticos son sustancias capaces de inhibir el crecimiento de los microrganismos o que los destruyen, y se utilizan para combatir las enfermedades infecciosas. A pesar de sus beneficios en la medicina, presentan algunos problemas importantes, tales como su limitada variedad, la interacción negativa potencial entre los diferentes tipos, etc. (Fothergill et al., 2006). Además, desarrollar nuevos antibióticos es un proceso largo y costoso (Cassell y Mekalanos, 2001).

Uno de los problemas primordiales que presentan los antibióticos, es el desarrollo de cepas con multirresistencia, lo cual incrementa la dificultad para tratar las enfermedades infecciosas. Los microrganismos constantemente se 
FIGURA 3. Aprobación de nuevos antibióticos por parte de la FDA. La cantidad de antibióticos aprobados disminuye con el tiempo.

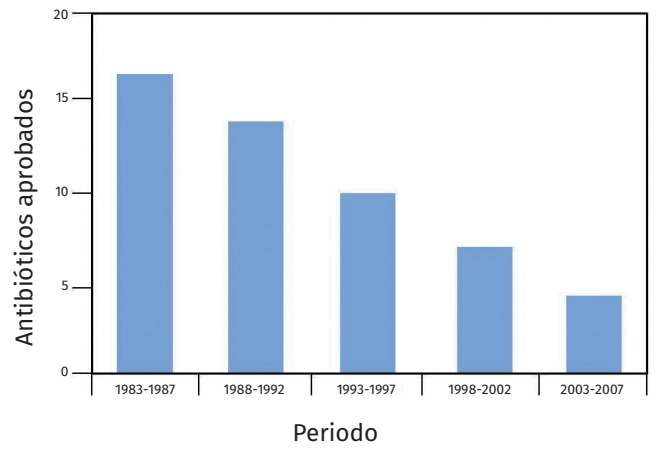

Fuente: Adaptado de Taubes, 2008.

adaptan y evolucionan para sobrevivir en condiciones nuevas o adversas. A pesar de la investigación científica para la modificación o desarrollo de nuevas sustancias antimicrobianas, no hay seguridad de que se continúen generando nuevos antibióticos al ritmo que se requiere (Taylor et al., 2002). En la figura 3, se muestra la cantidad de antibióticos aprobados por la FDA (Food and Drug Administration) por periodos de 5 años, desde 1983 hasta el 2007.

La resistencia contra antimicrobianos afecta a los países tanto industrializados como en desarrollo, provocando en los primeros un alto costo en los tratamientos, que llegan a ser prohibitivos en el caso de los países pobres. La proliferación de bacterias multirresistentes se asocia principalmente al mal manejo de los tratamientos antimicrobianos y a la alta tasa de infecciones nosocomiales (WHO, 2008). A estos problemas, se suman otros factores, tales como la aparición de focos epidémicos causados por desastres naturales o provocados por el hombre (inundaciones, guerras, hambruna, etcétera).

El resurgimiento de enfermedades infecciosas consideradas como controladas o erradicadas, conjugado con la ineficacia de los antibióticos, nos coloca en un reto científico muy importante para descubrir, diseñar o fabricar nuevas alternativas terapéuticas que nos permitan combatirlas de manera eficiente.

\section{Bionanotecnología y bionanomedicina}

La bionanotecnología es un área inherentemente interdisciplinaria. La bionanotecnología estudia los efectos y las interacciones que se presentan entre los materiales nanoestructurados y los sistemas biológicos. Investiga, por un lado, el efecto que provocan los nanomateriales en los seres vivos o sus componentes y, por otro, la influencia de los sistemas biológicos en las nanoestructuras, tales como su síntesis o modificación. La bionanotecnología 
aborda áreas que tienen un impacto potencial en la vida diaria. Existen muchas investigaciones orientadas a la biomedicina, producción y manejo de alimentos, y sobre la generación y optimización de la energía.

Hay muchas ramas de investigación y aplicaciones importantes de la bionanotecnología, entre las que destacan: la mejora de alimentos, producción y almacenamiento de energía, calidad del agua, tratamiento de enfermedades infecciosas, terapia génica, transporte de fármacos, tratamiento contra el cáncer, entre muchas otras. Sin duda alguna, uno de los sectores más estudiados es el relacionado con la salud, pues en conjunto con la bioingeniería, se preveé que la bionanotecnología tendrá un gran impacto en la investigación biomédica, es decir, en la bionanomedicina.

La bionanotecnología puede ayudar a mitigar el impacto de las enfermedades infecciosas. La bionanomedicina es un área de la bionanotecnología orientada a los aspectos biomédicos. En este sentido, la nanotecnología proporciona un panorama completamente nuevo, debido a la gran cantidad de materiales y procesos que se pueden generar y optimizar (Appasani, 2005).

\section{Nanomateriales de interés clínico}

Los nanomateriales son estructuras que se encuentran en un rango de tamaño de 1 a $100 \mathrm{~nm}$, aunque algunos autores incluyen a aquellos que miden incluso 1000 nm (Mohanraj y Chen, 2006; Buzea et al., 2007; Re et al., 2012). En estos tamaños, pueden cambiar algunas propiedades de la materia, por lo que las nanoestructuras poseen propiedades fisicoquímicas únicas (Kim et al., 2007). El tamaño es un parámetro de interés, debido a su impacto a nivel biológico. Las propiedades de los nanomateriales también dependen de los elementos que los constituyen y su forma (Mohanraj y Chen, 2006). En la figura 4, se muestran algunas nanopartículas de interés clínico.

Algunos nanomateriales tienen efectos contra los microrganismos patógenos. Los "nanoantibióticos" (Huh et al., 2011) son nanomateriales que muestran una actividad antimicrobiana o que elevan la efectividad y seguridad de los antibióticos. Las nanopartículas metálicas son un buen ejemplo de nanoantibióticos. En la tabla 1, aparecen diferentes nanomateriales con

FIGURA 4. Diferentes tipos de nanomateriales y nanopartículas. La mayoría de éstos poseen aplicaciones clínicas potenciales.
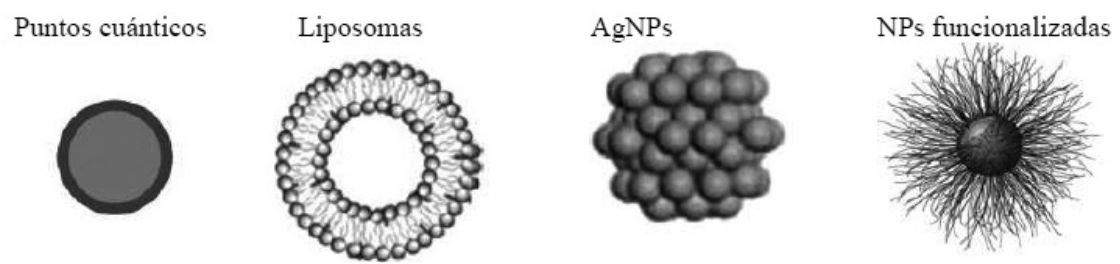

Fuente: Adaptado de “Nanoparticles for neuroimaging”, de Re et al., 2012; J. Phys. D: Appl. Phys., 45: 3. 
Tabla 1. Nanomateriales antimicrobianos. Se presentan diferentes nanomateriales que presentan aplicaciones potenciales relevantes.

\begin{tabular}{|l|l|}
\hline Nano-material & Aplicaciones potenciales: clínicas e industriales \\
\hline Nanopartículas de plata (AgNPs) & $\begin{array}{l}\text { Revestimiento de material quirúrgico, usos } \\
\text { clínicos y tratamiento de agua. Regeneración de } \\
\text { quemaduras, heridas y úlceras de pie diabético. }\end{array}$ \\
\hline Nanopartículas de zinc (ZnONPs) & $\begin{array}{l}\text { Artículos antibacteriales (cremas, geles, etc.); } \\
\text { revestimiento de material quirúrgico. }\end{array}$ \\
\hline $\begin{array}{l}\text { Nanopartículas de dióxido de } \\
\text { titanio ( } \mathrm{TiO}_{2} \mathrm{NPS} \text { ) }\end{array}$ & Agente antibacterial; purificador de agua y aire. \\
\hline Nanopartículas de oro (AuNPs) & Terapia fototérmica y uso antifúngico. \\
\hline $\begin{array}{l}\text { Nanopartículas de óxido nítrico } \\
\text { (NONPs) }\end{array}$ & Heridas infectadas y pie diabético. \\
\hline
\end{tabular}

Fuente: Adaptado de Huh et al., 2011.

propiedades antimicrobianas y se muestran sus aplicaciones potenciales, aunque es importante destacar que faltan muchos estudios todavía.

Entre los nanomateriales más estudiados por la bionanomedicina, se encuentran las nanopartículas metálicas, tales como las de plata (AgNPs), dióxido de titanio ( $\mathrm{TiO}_{2} \mathrm{NPs}$ ) y zinc ( $\mathrm{nnNPs}$ ). Las nanopartículas (NPs) metálicas presentan características importantes para las áreas microbiológicas y biomédicas (Sondi et al., 2004), aunque también muchas de ellas presentan riesgos potenciales para la salud y el ambiente. En la figura 5, se observa una comparación entre las nanopartículas y los sistemas biológicos.

\section{Ventajas y desventajas de los nanoantibióticos}

Los resultados de los estudios con nanoantibióticos muestran que éstos podrían ser una alternativa viable para combatir a los microrganismos patógenos. Las distintas nanopartículas tienen características variables que proporcionan diferentes propiedades físicas, químicas y biológicas, que pueden

FIGURA 5. Comparación de AgNPs de $10 \mathrm{~nm}$ y diferentes sistemas biológicos. Relación representativa de los tamaños entre una célula animal de $2 \mu \mathrm{m}$, una nanopartícula de $10 \mathrm{~nm}$ y una molécula de agua $\left(\mathrm{H}_{2} \mathrm{O}, 0.38 \mathrm{~nm}\right)$.

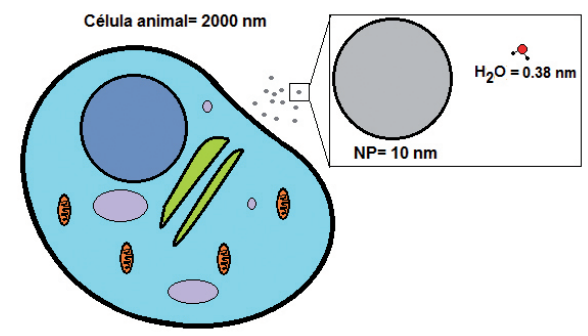

Fuente: Imágenes proporcionadas por Roberto Vazquez-Muñoz. 
ser aprovechadas en las áreas biomédicas. Algunas ventajas de las NPs son: baja citotoxicidad, menores costos de producción y la posibilidad de sobrepasar los mecanismos de resistencia (Allaker et al., 2000; Baker et al., 2005). Se ha reportado que algunos nanoantibióticos, como las nanopartículas de plata (AgNPs), tienen propiedades deseables en la clínica, tales como su capacidad antinflamatoria (Nadworny et al., 2008), la promoción de la regeneración de tejidos con quemaduras (Klansen, 2000) y su capacidad antiviral (Rogers et al., 2008).

Por otro lado, los nanomateriales presentan riesgos potenciales a la salud y al entorno. La exposición a los nanomateriales es inevitable, debido a que éstos son cada vez más comunes en la vida diaria. Así, la investigación en nanotoxicología busca evaluar los efectos de tales interacciones, pues se han reportado efectos tóxicos en la escala individual y ambiental (Ray et al., 2009). Entre los riesgos asociados a los nanomateriales, destacan las interacciones celulares y la toxicidad, el transporte y destino de las nanopartículas, su deposición y su movilidad, transformaciones redox, entre otros (Wiesner et al., 2006). En el caso de las nanopartículas metálicas, estás podrían dañar las membranas bacterianas a través de la posible liberación de iones metálicos, como la plata. Por lo que las células eucariotas (incluyendo las humanas) podrían verse afectadas de manera similar (Marambio-Jones y Hoek, 2010; Huh y Kwon, 2011). En la figura 6, se muestran posibles efectos de las nanopartículas metálicas sobre las células.

Es fundamental evaluar los parámetros de relevancia clínica, como la concentración inhibitoria, la concentración microbicida, el tiempo de exposición requerido, su estabilidad, los excipientes, posibles vías de aplicación, biocompatibilidad, índice de permeabilidad, entre otros. Por otro lado, se conocen de manera general los efectos de diferentes nanopartículas en los sistemas biológicos, se sabe muy poco acerca de sus mecanismos de acción. Se han propuesto diferentes mecanismos de acción para algunas NPs, tales como: la plasmólisis, la disrupción de los enlaces tiol y la liberación de iones metálicos (Feng et al., 2000).

Actualmente, se siguen desarrollando métodos de síntesis y aplicación de los nanomateriales, tema prioritario desde la perspectiva médica, económica y ambiental. También es primordial la evaluación del uso de nanomateriales en el contexto ecológico, incluyendo sus posibles efectos sobre la flora y la fauna del entorno, su biodisponibilidad y su deposición final.

\section{Consideraciones finales}

Considerando los aspectos generales sobre la importancia, impacto y aplicaciones potenciales de las nanopartículas metálicas, es fundamental estudiar las interacciones de los materiales nanoestructurados con los sistemas biológicos y el entorno. En el caso de la bionanomedicina, si se toman en cuenta los parámetros de producción y manejo, y estableciendo medidas de control, 
Figura 6. Diferentes efectos de las NPs metálicas más comunes: AgNPs, ZnONPs y $\mathrm{TiO}_{2} \mathrm{NPs}$

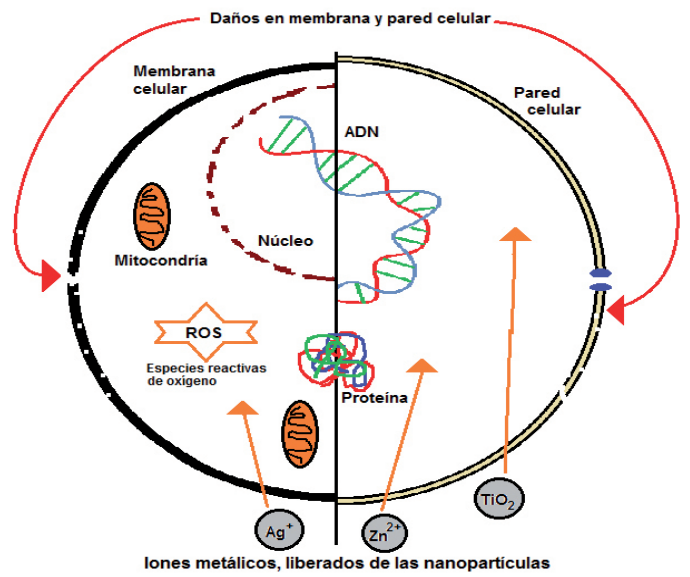

Fuente: Modificado de Huh et al., 2011.

estaremos en posibilidad de utilizar de una manera más amplia estos nanomateriales. Así, los nanoantibióticos podrían ser una alternativa al uso de los antibióticos en diversos campos o áreas de interés médico y socioeconómico, tales como la clínica, la veterinaria, la agricultura y la ganadería.

\section{Bibliografia}

Ahamed, M. (2011) Toxic response of nickel nanoparticles in human lung epithelial A549 cells. Toxicology in Vitro, 25: 930-936.

Allaker, R. Ren, G. (2008) Potential impact of nanotechnology on the control of infectious disease. Trans. R. Soc. Trop. Med. Hyg. 102(1): 1-2.

Appasani, K. (2005) BioNanoMedicine: A nanotechnology platform for the 21st century. Expert Rev. Mol. Diagn., 5(6): 839-40.

Baker, C. Pradhan, A., Pakstis, L., Pochan, D., Shah, S. (2005) Synthesis and antibacterial properties of silver nanoparticles. J Nanosci Nanotechnol, 5: 244-249. DOI:10.1166/ jnn.2005.034.

Buzea, C., Pacheco, I. y Robbie, K. (2007). Nanomaterials and nanoparticles: Sources and toxicity. Biointerphases, 2(4): MR17-MR172.

Cassell, G. y Mekalanos, J. (2001) Development of antimicrobial agents in the era of new and reemerging infectious diseases and increasing antibiotic resistance. JAMA, 285(5): 601-605. DOI:10.1001/jama.285.5.601.

Centers for Disease Control and Prevention (CDC) (2010). Recuperado: 10 de febrero del 2014 <http://www.cdc.gov/parasites/about.html>.

Clancy, C. y Nguyen, M. (2012) Systemic candidiasis: Candidemia and deep-organ infections. En Calderone, R.A. y Clancy, C.J. (eds.) Candida y candidiasis (pp. 387401). Washington DC, ASM Press.

Eggimann, P. Garbino, J. y Pittet, D. (2003) Epidemiology of Candida species 
infections in critically ill non-immunosuppressed patients. Lancet Infect., Dis., 3: 685-702.

Feng, Q. Wu, J., Chen, G., Cui, F., Kim. y Kim, J. (2000) A mechanistic study of the antibacterial effect of silver ions on E. coli and Staphylococcus aureus. J Biomed Mater Res, 52: 662-8.

Fonkwo, P. (2008) Pricing infectious disease. The economic and health implications of infectious diseases. EMBO Rep., 9(1): S13-S17. doi: 10.1038/embor.2008.110.

Fothergill, A.W. Rinaldi, M.G. y Sutton, D.A. (2006) Antifungal susceptibility testing. Infect Dis Clin N Am, 20: 699-709.

Fridkin, S.K. (2005) The changing face of fungal infections in health care settings. Clin. Infect. Dis. 41: 1455-1460.

Huh y Kwon, Y. (2011) Nanoantibiotics: A new paradigm for treating infectious diseases using nanomaterials in the antibiotics resistant era. J. Control Release, 156: 128-145. DOI: 10.1016/j.jconrel.2011.07.002.

IBNAM: Institute of Bionanotecnology in Medicine. Recuperado: 10 de febrero del 2014, de <http://www.ibnam.northwestern.edu/about.html>.

Klasen, H. (2000) (a). Historical review of the use of silver in the treatment of burns. I. Early uses. Burns, 26: 117-138.

Marambio-Jones, C. y Hoek, E.M.V. (2010) A review of the antibacterial effects of silver nanomaterials and potential implications for human health and the environment. J Nanopart Res. 12: 1531-1551. DOI 10.1007/s11051-010-9900-y.

Martin, G.S., Mannino, D.M., Eaton, S., y Moss, M. (2003) The epidemiology of sepsis in the United States from 1979 through 2000. N. Engl. J. Med., 348: 15461554.

Mcneil, M., Nash, S., Hajjeh, R., Phelan, M., Conn. L., Plikaytis, B., y Warnock, D. (2001) Trends in mortality due to invasive mycotic diseases in the United States, 1980-1997. Clin. Infect. Dis. 33: 641-647.

Med-health (2014) Recuperado: 10 de febrero del 2014, de <http://www.medhealth.net/Protozoan-Diseases.html>.

Mohanraj, V. y Chen, Y. (2006) Nanoparticles - A review. Tropical Journal of Pharmaceutical Research, 5(1): 561-573

Nadworny, P., Wang, J., Tredget, E. y Burrell, R. (2008) Anti-inflammatory activity of nanocrystalline silver in a porcine contact dermatitis model. Nanomedicine, 4: 241-251.

National Institute of Allergy and Infectious Diseases (NIAID) (2009) Understanding microbes in sickness and in health. NIH Publication No. 09-4914, p 12. Recuperado: 10 de febrero de 2014, de <http://www.niaid.nih.gov/topics/microbes/ documents/microbesbook.pdf $>$.

National Library of Medicine, National Institutes of Health (NLM) (2013) Infectious diseases. Recuperado: 10 de febrero, de <http://www.nlm.nih.gov/medlineplus/infectiousdiseases.html>.

National Library of Medicine, National Institutes of Health (NLM) (2014) Recuperado: 10 de febrero, de <http://www.nlm.nih.gov/medlineplus/viralinfections. html>. 
NIAID: National Institute of Allergy and Infectious Diseases. Understanding Microbes in Sickness and in Health. NIH Publication No. 09-4914, p. 12. Recuperado: 10 de febrero del 2014, de <http://www.niaid.nih.gov/topics/microbes/documents/microbesbook.pdf $>$.

Pfaller, M. y Diekema, D. (2006) Epidemiology of invasive candidiasis: A persistent public health problem. Clinical Microbiology Reviews, 20: 133-63.

Ray, P.C., Yu, H. y Fu, P.P. (2009) Toxicity and environmental risks of nanomaterials: Challenges and future needs. J Environ Sci Health C Environ Carcinog Ecotoxicol Rev., 27(1): 1-35. doi: 10.1080/10590500802708267.

Re, F., Moresco, R. y Masserini, M. (2012) Nanoparticles for neuroimaging. J. Phys. D: Appl. Phys., 45073001.

Rogers, J., Parkinson, C., Choi, Y., Speshock, J. y Hussain, S. (2008) A preliminary assessment of silver nanoparticle inhibition of monkeypox virus plaque formation. Nanoscale Res Lett, 3: 129-133.

Scott, D. (2009) The direct medical costs of healthcare-associated infections in U.S. hospitals and the benefits of prevention. National Center for Preparedness, Detection, and Control of Infectious Diseases. Recuperado: 3 de julio de 2013, de <http:// www.cdc.gov/hai/pdfs/hai/scott_costpaper.pdf >.

Sondi, I. y Salopek-Sondi, B. (2004) Silver nanoparticles as antimicrobial agent: A case study on E. coli as a model for Gram-negative bacteria. J Colloid Interface Sci, 275: 177-182. DOI:10.1016/j.jcis.2004.02.012.

Talyor P., Stapleton P. y Luzio J. (2002) New ways to treat bacterial infections. Drug Discov. Today, 7(21): 1086-1091.

Ulrich, D. y Hacker, J. (2001) Whole genome plasticity in pathogenic bacteria. Current Opinion in Microbiology, 4: 550-557.

UNICEF/WHO (2009) Diarrhoea: Why children are still dying and what can be done. The United Nations Children's Fund (UNICEF)/World Health Organization (WHO), ISBN 978-92-806-4462-3 (UNICEF).

Walsh, T., Groll, A., Hiemenz, J., Flemming, R., Roilides, E. y Anaissie, E. (2004) Infections due to emerging and uncommon medically important fungal pathogens. Clin. Microbiol. Infect., 10(1): 48-66.

WHO (2013) <http://www.who.int/mediacentre/factsheets/fs110/en/>.

Wiesner, M., Lowry, G., Álvarez, P., Dionysiou, D., Biswas, P. (2006) Assessing the risks of manufactured nanomaterials. Environmental Science y Technology. <http://pubs.acs.org/doi/pdf/10.1021/es062726m>.

World Health Organization (WHO) (1997) The world health report 1997 - conquering suffering, enriching humanity. Recuperado: 10 de febrero de 2014, de <http:// www.who.int/whr/1997/media_centre/50facts/en/>.

World Health Organization, WHO (2000) Millennium Development Goals (MDGs). Recuperado 16 de julio del 2013, de: <http://www.who.int/topics/millennium_development_goals/en/>.

World Health Organization (WHO) (2008) What are the International Health Regulations? Recuperado: 10 de febrero de 2014, de <http://www.who.int/features/ qa/39/en/index.html>. 